The Ascent of Bitcoin: Bibliometric Analysis of Bitcoin Research

\author{
Ahmet Faruk Aysan \\ Hamad Bin Khalifa University, \\ Professor \& Program Coordinator, PhD in Islamic Finance and Economy \\ College of Islamic Studies, Qatar Foundation \\ Orcid: 0000-0001-7363-0116 \\ aaysan@hbku.edu.qa \\ Hüseyin Bedir Demirtaş \\ Bogazici University, Department of Economics, Istanbul, Turkey \\ huseyin.demirtas1@,boun.edu.tr \\ Mustafa Saraç \\ Bogazici University, Department of Management, Istanbul, Turkey \\ mustafa.sarac@boun.edu.tr
}




\title{
The Ascent of Bitcoin: Bibliometric Analysis of Bitcoin Research
}

\begin{abstract}
Bitcoin, as the first decentralized cryptocurrency, pioneers the cryptocurrency markets, both in terms of market capitalization and scientific interest. In this paper, we performed a comprehensive bibliometric study of the Bitcoin-related literature. Using the Scopus database, we created a sample that comprises 4495 documents written in the 2011-2020 period. Furthermore, we provided insights about dimensions such as the change in the number of publications over the course of years, the main research areas, types of published documents, most important platforms and sources of Bitcoin publications, highly cited studies, productive authors, author's countries, and finally main funders of Bitcoin-related research. Lastly, our bibliometric study manifests the current state and future path of Bitcoin literature from distinct perspectives.
\end{abstract}

Keywords: Cryptocurrencies; Blockchain; Bitcoin; Scopus; VOSviewer; Bibliometrics; Research

JEL codes: G19; E4 


\section{The Ascent of Bitcoin: Bibliometric Analysis of Bitcoin Research}

\section{Introduction}

Cryptocurrency is a digital asset that enables people to transact with each other since it could be used as a medium of exchange. Furthermore, cryptocurrency owners store their assets in a digitally distributed ledger in a system of a decentralized network that records transactions of various users. Cryptocurrencies are not issued by central authorities; therefore, their value mostly comes from the scale of participation within the market. Still, value formation and fluctuations of cryptocurrencies are hotly debated topics in the literature (Aysan et al. 2021, 2020, 2019; Hayes, 2017; Nadler \& Guo, 2020, Akyildirim et al. 2021). Bitcoin is the most commonly used cryptocurrency among the various cryptocurrencies in the market. Its market cap exceeds 600 billion dollars as of January 2021 (Coinmarket, 2021). In 2009, an anonymous writer, named Satoshi Nakamoto, published a paper titled "Bitcoin: A Peer-to-Peer Electronic Cash System" that discussed the possibility of establishing a new digital currency (that is not controlled or issued by a central authority) by creating a decentralized network. England and Fratrik (2018) argue that there are four reasons that drove people to use Bitcoin widely and increase their interest in it: (a) advances in cryptography, (b) infringements on individual privacy due to the events that occurred after 2001, (c) inflation expectations due to the massive stimulus packages and reserve pumping into the markets to downshift the impacts of the Global Financial Crisis (GFC) in 2008, and (d) increasing dissatisfaction with banks and other large financial institutions because of the policies that lead to the subprime mortgage crisis and then worldwide crisis. Further, Bouri et al. (2017a, 2017b) and Bouri et al. (2019) discuss the usage of Bitcoin as a hedge against global uncertainty and comparing it with traditional investment instruments such as gold and commodities. Bitcoin has experienced a massive price jump up to $\$ 20,000$ in 2017 , however, its value started to decrease under $\$ 10,000$ s afterward. Nevertheless, Bitcoin prices skyrocketed in the last quarter of 2020. Its price increased from $\$ 10,169.57$ in August 2020, up to its all-time high of $\$ 61,712$ in March 2021. Figure 1 plots the historical Bitcoin prices between 2014-2021. 


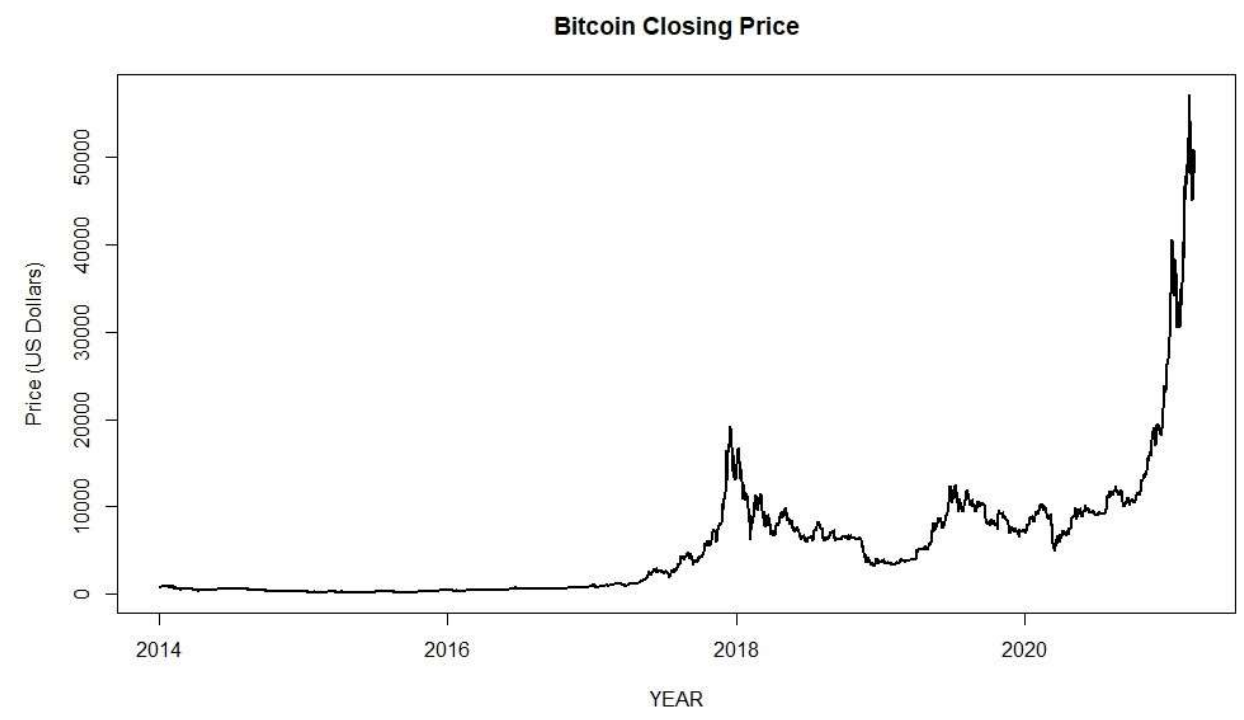

Figure 1: The course of Bitcoin prices over the years

Today, scientific papers are collected in large databases such as Scopus, Web of Science, and ScienceDirect, etc. This enables evaluating various aspects of such papers, e.g. number of authors, keywords, subject, citation numbers, institutional collaboration, and so on. Institutions, by using these sources, can obtain valuable information about individual and aggregate impact. It may also facilitate new researchers of a discipline to comprehend the ramifications of a subject, emerging trends, and its course in time. In this regard, it differs from traditional literature surveys. Such indexing services are an important input of the evaluation process in academia and give clues about the retrospect and even prospect of the existing ideas.

Bibliometric analysis is assumed to have been introduced by Price (1965) paper named "Networks of Scientific Papers" to identify relationships between articles based on their citation numbers. Bibliometrics is a quantitative technique used to estimate, analyze, and visualize the construction of scientific fields (Aysan et. al 2021, Koskinen et al., 2008). It is engaged for the purpose of describing the expansion of the desired field in a particular area of knowledge (H. Liu, Zhang, \& 
Yang, 2018). Further, it entails making an evaluation of publications such as the impact factor, citations, publishers, and countries of publication (Lee, 2019; Docampo \& Cram, 2019; Iefremova, Wais, \& Kozak, 2018).

- Authors can ascertain the impact of their publications, findings, and research;

- Institutions can evaluate publications and measure their impact and performance;

- Researchers can foresee future research trends and the prospective research subjects;

- Analysts can assess the growing strands of literature and body of knowledge as a whole.

In social sciences, methodologies such as bibliometric analysis (quantitative) and content analysis (qualitative) are gaining popularity in academic circles. Although such methods are still underutilized in business, economics and finance research it is possible to identify several studies of this kind (Zamore et. al, 2018; Helbing, 2019). For instance, Bonilla et al. (2015) scrutinize the academic research that flourished in Latin America in the economics discipline between the years 1994 and 2013. Andrikopoulos et al. (2016) developed a bibliometric analysis of the economics. In his study, he reviewed the first 40 years of the Journal of Econometrics and focused on collaboration formations. He further looked up how econometric methods internationalized in the discipline. CastilloVergara et. al (2018) documented the evolution of creativity in the field of business. Wei (2019) is another study in which economic journals are viewed from a bibliometric lens. Further, Costa et al. (2019), tried to unravel the course of Behavioral Economics and Behavioral Finance in the field of economics by utilizing bibliometric methods. Korom (2019), the author constructs co-citation networks and obtains thematic clusters to analyze the thematic overlap between economic and sociological approaches to wealth inequality subject. 
The aim of our paper is to analyze and assess the metadata of all the papers indexed in the Scopus database, whose subject is "Bitcoin". Our ultimate goal is to present a detailed picture of academic studies that are focusing on different aspects of Bitcoin. We provide insights on different categories such as the change in the number of publications over the course of years, the main research areas, the types of published documents, most important platforms and sources of Bitcoin publications, highly cited articles, most productive authors, author's countries, and finally main funders of Bitcoin-related research. Our paper differs from the relevant bibliometrics studies in the literature in two respects; it employs the biggest data by analyzing 4495 documents, and it is the most up-to-date bibliometric study of Bitcoin-related research in which the Scopus database is utilized. With our study, we hope to shed light on future research trends and the prospective research subjects of Bitcoin-related academic output. Moreover, we hope that it will be beneficial in the assessment of the expanding strands of Bitcoin-related literature and the body of knowledge as a whole.

\section{Literature Review of Bibliometric Studies on Bitcoin}

There are several articles that are concerned with the bibliometric analysis of academic studies in which Bitcoin is the main subject. In the relevant literature, it is possible to see papers that comprise different time periods and utilizing different databases and tools. The first of the papers that carry out a bibliometric analysis of Bitcoin research is Liu (2016). In his paper, Liu uses the Scopus database from which he collected 253 articles published as of July 2016. He models the Bitcoin research in a commonly used macroeconomic analysis structure-PEST analysis (political, economic, social, and technological) framework. He concludes that current Bitcoin research is separated into three categories: technological, economic, and financial, as well as legal and regulatory. He further suggests that there is a big research gap to be filled in the social domain (J. Liu et al., 2016). Holub and Johnson (2017), collect 4429 papers from different databases such as Web of Science, SpringerLink, Scopus, EconPapers, SSRN, and public working papers repositories such as ArXiv or SSRN. They use 1206 of these papers for a 
comprehensive analysis. Another paper Merediz-Solà \& Bariviera (2019), utilized the metadata they acquired from the Web of Science Core Collection that included 1162 papers in total for the years 2012-2019. Their paper can be considered as an extension to Holub and Johnson (2017), adding almost two-year data. Unlike Holub and Johnson (2017), which uses multiple data sources, they restrict their research solely to the Web of Science database. They analyze the documents in their dataset from various standpoints such as main research areas, fields, geographic distribution, publication sources, main keywords, and degree of concentration, and so on. In addition, the authors calculate the normalized entropic concentration index for the distribution of authors, sources, countries, research areas, and citations in the Bitcoin literature. They observe that paper citations are very concentrated and only 58 out of the 1162 papers in their sample account for $50 \%$ of all citations in the literature (Merediz-Solà \& Bariviera, 2019). The last of the papers with regards to the bibliometric study of Bitcoin research is Ramona \& Cristina \& Raluca (2019). In the paper, the authors try to analyze the growing scientific literature on Bitcoin published between 2012 and 2019 using the Web of Science Core Collection. The sample they use in the paper includes 911 documents in total. They also provide a knowledge area map that identifies and evaluates the links between authors and countries distribution, the conceptual structure of the field, the structure and connections of most cited papers and journals (Ramona, Cristina, \& Raluca, 2019). 
3 However, Bitcoin is not the only subject of bibliometric studies when it comes to cryptocurrencies. Since Bitcoin, as the most renowned cryptocurrency, draws its strength from the underlying blockchain technology, it would also be fruitful to investigate the bibliometric studies regarding blockchain to gain insights for the possible research trajectories of the Bitcoin-related studies. On a broader scale, there are several bibliometric studies regarding blockchain technology. To mention but a few, Firdaus et al. (2019), for instance, conducted a comprehensive study on the blockchain by using the Scopus database. The findings indicate the trend of blockchain technology in different areas as well as different activity levels in terms of publications and research collaborations for countries. Further, their research highlighted the utilization and consensus algorithms in blockchain research (Firdaus et al., 2019). 
Furthermore, recently, Guo et al. (2021)

conducted a bibliometric study on the

blockchain. Their research clarifies the

development trends and current domains in the

field using $\mathbf{3 8 2 6}$ articles that were published from

2013 to 2020. The findings indicate productive

and influential authors, institutions, journals,

and countries. Further, their study indicated

areas that future blockchain studies should

concentrate on: (a) management, (b) blockchain

technology, (c) energy, (d) machine learning, and

(e) smart home (Guo et al., 2021).Data and Methodology

In our paper, we constructed the sample from the Scopus database. Launched in 2004, Scopus is the largest abstract and citation database consisting of peerreviewed publications that can be used to monitor, analyze, and visualize the research and the literature. The aim is to keep track of scientific research effectively and efficiently. Scopus database covers over 24,500 active titles from more than 5,000 publishers all over the world in the fields of science, technology, medicine, and social sciences, as well as arts and humanities. Breakdown of the content of the Scopus database in terms of categories is presented in Table 1.

Table 1: Scopus content coverage by subject area 


\begin{tabular}{lcc}
\hline Subject Area & Number of Titles & Percentage \\
\hline Life Sciences & 4,883 & $16 \%$ \\
Health Sciences & 7,468 & $25 \%$ \\
Physical Sciences & 8,102 & $27 \%$ \\
Social Sciences & 9,692 & $32 \%$ \\
\hline
\end{tabular}

Source: Scopus Global Research Factsheet 2019, www.elsevier.com

In our search, we, by using the Scopus database, created a dataset by searching the word 'bitcoin' within three dimensions: (a) article titles, (b) abstract, and (c) keywords. The query string identified nearly 4700 documents in the Scopus database. Among these documents, some of them were listed without authors, some of them were non-academic, some of them were in languages other than English, and some of them were duplications. After removing these documents, there are 4495 documents left that are ready to be scrutinized. We also did not include studies published in the year 2021 in our sample to have comparability between different years. In the end, 4495 documents which are obtained from Scopus become the subject of this study. Although Scopus is a dynamic database that is subject to retrospective updates, we provide a full-fledged snapshot of the Bitcoin research between the years 2011 and 2020 through the examination of different categories and parameters.

\section{Results}

The number of publications by year is displayed in Table 2 . We can observe that at the initial stages, such as the first three years of the sample, the relative increase exceeds $100 \%$, even reaching $300 \%$. After the first three years, the number of yearly publications started to decrease, the growth rate in publications plunged to $9 \%$. However, interest in Bitcoin increased once again, as the number of publications showed an increasing trend after 2017. The rise in the number of publications, probably, caused by the massive increase in Bitcoin prices in 2017. In 2017, Bitcoin prices skyrocketed from $\$ 1,000$, at the beginning of 2017 , to nearly $\$ 20,000$ at the end of the year. The yearly growth rate showed a decline for the first time in history in 2020. The decreasing growth rate could be a sign of the possibility that research in this field is consolidating. Even if the number of 
publications experienced a decline in 2020, still, almost 50\% of all publications about the topic were published, in the last two years. Although Bitcoin-related research experienced a decline for the first time in years, the number of articles published is still in an increasing trend, as Figure 2 depicts. A decrease in the growth rate of total publications and preserving the increasing trend in the number of articles published could be a sign of the impact of COVID-19 on bitcoin-related research. As Table 4 indicates bitcoin publications are largely composed of either conference papers or articles. Conference papers refer to articles that are written to be accepted to a conference. In this sense, conference organizations might have been affected by the COVID-19 since many meetings were canceled in 2020, due to the COVID-19 infection risk. The declined trend in the growth rate could be reversed in the following years, as more entities started to accept Bitcoin as a payment method and more central banks around the world work on introducing digital currencies and new regulations governing the cryptocurrency markets.

Table 2: Number of publications by year

\begin{tabular}{lccc}
\hline Year & \# of Articles & Yearly Growth Rate & \% Total Publications \\
\hline 2011 & 3 & - & $0.07 \%$ \\
2012 & 12 & $300 \%$ & $0.27 \%$ \\
2013 & 33 & $175 \%$ & $0.73 \%$ \\
2014 & 128 & $288 \%$ & $2.85 \%$ \\
2015 & 210 & $64 \%$ & $4.67 \%$ \\
2016 & 229 & $9 \%$ & $5.09 \%$ \\
2017 & 416 & $82 \%$ & $9.25 \%$ \\
2018 & 832 & $100 \%$ & $18.51 \%$ \\
2019 & 1321 & $59 \%$ & $29.39 \%$ \\
2020 & 1311 & $-1 \%$ & $29.17 \%$ \\
\hline
\end{tabular}

Source: Authors' calculations based on Scopus data set 


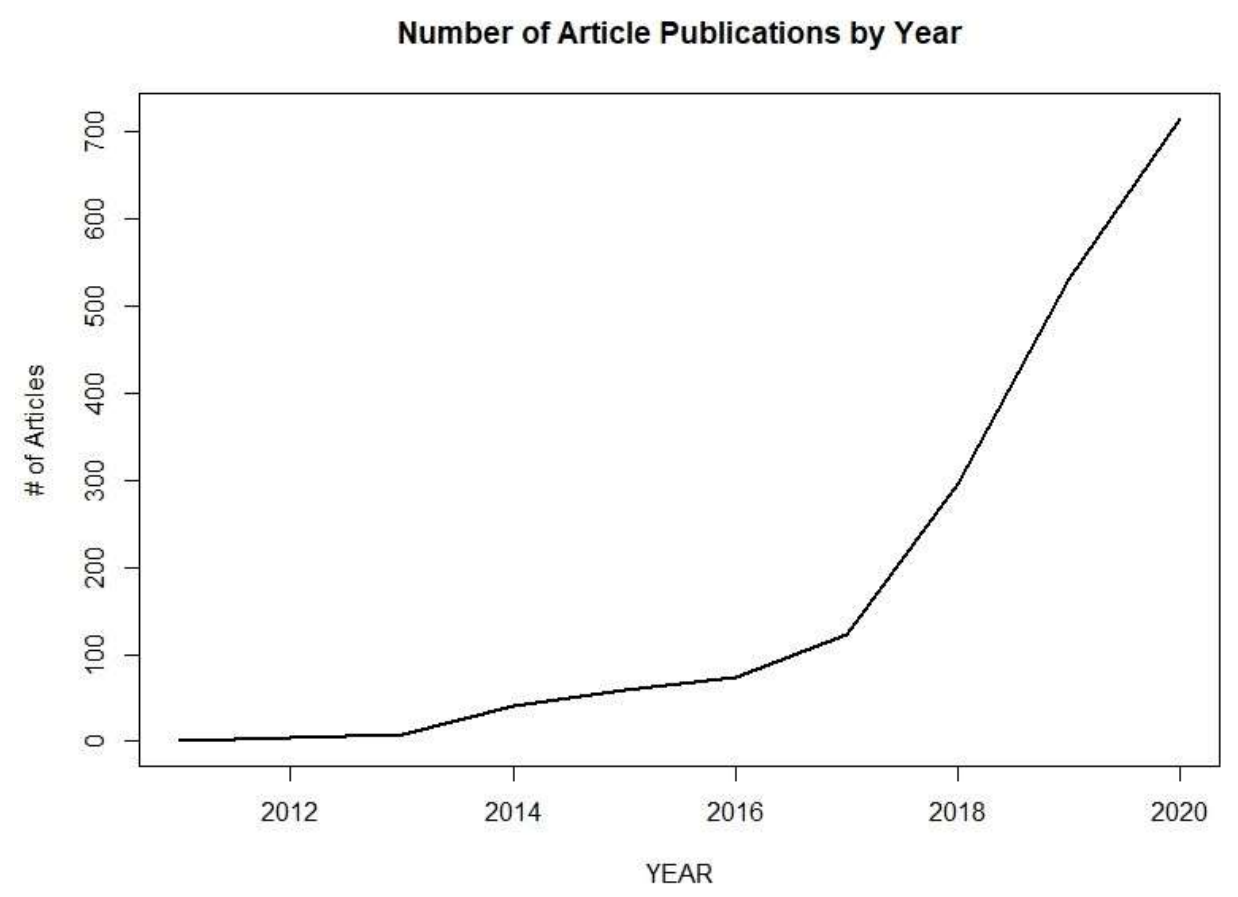

Figure 2: Number of article publications by year

Table 3: Main research areas of Bitcoin studies

\begin{tabular}{lccc}
\hline Research Areas & \# of Publications & \% in Total Publications & Cumulative Percentage \\
\hline Computer Science & 2881 & $33.40 \%$ & $33.40 \%$ \\
Engineering & 1140 & $13.20 \%$ & $46.60 \%$ \\
Mathematics & 968 & $11.20 \%$ & $57.80 \%$ \\
Economics, Econometrics and Finance & 876 & $10.10 \%$ & $67.90 \%$ \\
Business, Management and Accounting & 692 & $8.00 \%$ & $76.00 \%$ \\
Decision Sciences & 595 & $6.90 \%$ & $82.80 \%$ \\
Social Sciences & 507 & $5.90 \%$ & $88.70 \%$ \\
Physics and Astronomy & 221 & $2.60 \%$ & $91.30 \%$ \\
Materials Science & 132 & $1.50 \%$ & $92.80 \%$ \\
Energy & 127 & $1.50 \%$ & $94.30 \%$ \\
\hline
\end{tabular}

Source: Authors' calculations based on Scopus data set

Scopus database assigns some of the papers into more than one research area. Therefore, 4495 documents in our sample were assigned into 8633 research areas, 
in total. In Table 3, we show the main research areas of Bitcoin studies. Although the Scopus database classifies related papers under twenty-five subject categories, most of the Bitcoin-related papers are concentrated on certain research areas. The top three research areas Computer Science, Engineering, and Mathematics, respectively. Computer Science takes the lead with constituting $33.40 \%$ of total publications. In fact, these top three research areas constitute almost $60 \%$ of all papers. Furthermore, Economics, Finance, and Business Studies follow those top three research subjects. The top ten research areas indicated in Table 3 constitute a cumulative percentage of $94.30 \%$ of total publications in Bitcoin-related papers.

Table 4: Bitcoin publications by document type

\begin{tabular}{lcc}
\hline Document Type & \# of Publications & \% of Total Publications \\
\hline Conference Paper & 2097 & $47 \%$ \\
Article & 1875 & $42 \%$ \\
Conference Review & 175 & $4 \%$ \\
Book Chapter & 156 & $3 \%$ \\
Review & 92 & $2 \%$ \\
Book & 24 & $1 \%$ \\
\hline
\end{tabular}

Source: Authors' calculations based on Scopus data set

Bitcoin-related studies are published in different types of documents. In Table 4, we showed the Bitcoin publications by document types. Summary statistics show that most of the documents are concentrated in two document type categories. Most of the publications are published either as a Conference Paper or an Article. These two document types constitute $47 \%$ and $42 \%$ of total publications, respectively. 
Table 5: Most important sources of Bitcoin publications

\begin{tabular}{|c|c|c|}
\hline Source Title & \# of Articles & Type \\
\hline Lecture Notes in Computer Science & 437 & Conference Proceedings \\
\hline Finance Research Letters & 112 & Journal \\
\hline ACM International Conference Proceeding Series & 96 & Conference Proceedings \\
\hline Advances in Intelligent Systems and Computing & 66 & Conference Proceedings \\
\hline IEEE Access & 63 & Journal \\
\hline Communications in Computer and Information Science & 59 & Conference Proceedings \\
\hline Physica A: Statistical Mechanics and its Applications & 56 & Journal \\
\hline Proceedings of The ACM Conference on Computer and Communications Security & 51 & Conference Proceedings \\
\hline Economics Letters & 47 & Journal \\
\hline Ceur Workshop Proceedings & 43 & Conference Proceedings \\
\hline Research in International Business and Finance & 31 & Journal \\
\hline Future Generation Computer Systems & 28 & Journal \\
\hline International Review of Financial Analysis & 28 & Journal \\
\hline Lecture Notes in Business Information Processing & 27 & Conference Proceedings \\
\hline Economist (United Kingdom) & 23 & Newspaper \\
\hline Plos One & 23 & Journal \\
\hline Applied Economics Letters & 22 & Journal \\
\hline Quarterly Review of Economics and Finance & 20 & Journal \\
\hline Journal of Physics Conference Series & 18 & Conference Proceedings \\
\hline Applied Economics & 16 & Journal \\
\hline
\end{tabular}

Source: Authors' calculations based on Scopus data set

Table 5 presents summary statistics for the main sources of the studies concerning Bitcoin. Among the sources two particularly stand out: Lecture Notes in Computer Sciences and Finance Research Letters. Lecture Notes in Computer Science is a series of computer science books published by Springer since 1973. It is an important platform for the computer science discipline since new developments in computer science and information technology research and teaching are published in these series. Two sub-series are Lecture Notes in Artificial Intelligence and Lecture Notes in Bioinformatics. Lecture Notes in Computer Sciences is followed by Finance Research Letters and ACM International Conference Proceedings Series. The second one, Finance Research Letters, is a journal that accepts submissions from all areas of finance. It is worth noting that majority of the studies in Bitcoin research are published either as conference proceedings series or journal papers. Furthermore, as can be seen, the main sources for these studies are from two prominent fields: computer engineering and 
economics (in particular finance). Finance Research Letters and Economic Letters are two leading economics journals in Bitcoin research. Having sources from computer science, physics and economics reflect the interdisciplinarity of Bitcoin research.

\section{Table 6: Highly cited articles (in descending order)}

\begin{tabular}{llcc}
\hline Authors and Publication Years & \multicolumn{1}{c}{ Document Title } & \# of Citations & Research Area \\
\hline Zyskind et al. (2015) & Decentralizing privacy: Using blockchain to protect personal data & 922 & Computer Science \\
Zheng et al. (2017) & An Overview of Blockchain Technology: Architecture Consensus and Future Trends & 829 & Computer Science \\
Khan and Salah (2018) & IoT security: Review blockchain solutions and open challenges & 552 & Computer Science \\
Yli-Huumo et al. (2016) & Where is current research on Blockchain technology? - A systematic review & 573 & Multidisciplinary \\
Tschorsch and Scheuermann (2016) & Bitcoin and beyond: A technical survey on decentralized digital currencies & 550 & Engineering \\
Luu et al. (2016) & Making smart contracts smarter & 551 & Computer Science \\
Androulaki et al. (2018) & Hyperledger Fabric: A Distributed Operating System for Permissioned Blockchains & 538 & Computer Science \\
Ben-Sasson et al. (2014) & Zerocash: Decentralized anonymous payments from Bitcoin & 538 & Computer Science \\
Eyal et al. (2016) & Bitcoin-NG: A scalable blockchain protocol & 467 & Computer Science \\
Gervais et al. (2016) & On the security and performance of Proof of Work blockchains & 442 & Computer Science \\
Decker and Wattenhofer (2013) & Information propagation in the Bitcoin network & 425 & Engineering \\
Bonneau et al. (2015) & SoK: Research perspectives and challenges for Bitcoin and cryptocurrencies & 424 & Computer Science \\
Bohme et al. (2015) & Bitcoin: Economics technology and governance & 420 & Economics, Econometrics and Finance \\
Garay et al. (2015) & The Bitcoin backbone protocol: Analysis and applications & 417 & Computer Science \\
Meiklejohn et al. (2013) & A fistful of Bitcoins: Characterizing payments among men with no names & 402 & Computer Science \\
\hline
\end{tabular}

Source: Authors' calculations based on Scopus data set

Table 6 shows the list of the 15 highly cited articles in descending order and research areas of each. Highly cited or top-cited papers are works that possibly have had a great influence in the field with their scientific quality and excellence or because they pioneered the research in the field when Bitcoin was still a quandary in the scientific circles. At the first glance, Computer Science is the most popular research area with its share of almost 80 percent in the top 15 highly cited papers in our data set. Interestingly, even though Bitcoin is primarily a financial instrument, only one of the top 15 highly cited papers is classified under the Economics, Econometrics and Finance. Furthermore, highly cited papers signal the future trends in the research paths in the field. Zyskind et al. (2015) discuss the recent issues of surveillance, security breaches, and data privacy in which third-parties collect. In their paper, the authors offer a new decentralized personal data management system that ensures users own and control their data by implementing an access-control protocol in the system. Lastly, they compare the current financial system that Bitcoin markets use and their decentralized system (Zyskind et al., 2015). On the other hand, Khan and Salah (2018) analyze the relationship between Blockchain technology and its usage for Bitcoin and current IoT security problems (Khan \& Salah, 2018). Yli-Huumo et al. (2016) investigate the current trends in Blockchain technology. The results show that the focus is 
mostly on Bitcoin as most of the research, i.e. $80 \%$, deals with the Bitcoin system. Tschorsch and Scheuermann (2016) investigate the impact of the building blocks of Bitcoin and its applications in many areas. They showed that many key ideas are likewise applicable in various other fields so that their impact reaches far beyond than where Bitcoin had reached. Ben-Sasson et al. (2014) discuss the possibility of a payment system with digital currencies. They constructed a fullfledged ledger-based digital currency with strong privacy guarantees. Further, their results leveraged recent advances in zk-SNARKs. They develop the Zero cash, a practical instantiation of their DAP scheme construction, in which transactions are less than $1 \mathrm{kB}$ and take under $6 \mathrm{~ms}$ to verify orders of magnitude more efficient than the less-anonymous Zero coin and competitive with plain Bitcoin (Sasson et al., 2014). Meiklejohn et al. (2013) investigate the Bitcoin market and its usage as a payment system in the future, notwithstanding mentioning Bitcoin applications for criminal and fraudulent activities.

Table 7: Most productive authors of Bitcoin studies

\begin{tabular}{lc}
\hline Author's Name & \# of Articles \\
\hline Bouri, E. & 39 \\
Kiayias, A. & 26 \\
Roubaud, D. & 26 \\
Corbet, S. & 23 \\
Lucey, B. & 18 \\
Miller, A. & 18 \\
Zohar, A. & 17 \\
Bartoletti, M. & 16 \\
Gupta, R. & 15 \\
Wattenhofer, R. & 14 \\
\hline
\end{tabular}

Source: Authors' calculations based on Scopus data set

Table 7 presents the most productive authors of Bitcoin-related articles. The first one E. Bouri with 39 published articles, followed by A. Kiayias and D. Roubaud 
who are also produced more than 25 articles until now. S. Corbet, B. Lucey and A. Miller are next other influential authors.

Table 8: Top 5 funding sponsors

\begin{tabular}{lcc}
\hline Funding Sponsor & \# of Publications & \% of Total Funded Publications \\
\hline National Natural Science Foundation of China & 265 & $16.7 \%$ \\
National Science Foundation & 137 & $8.6 \%$ \\
National Research Foundation of Korea & 54 & $3.4 \%$ \\
Fundamental Research Funds for the Central Universities & 50 & $3.1 \%$ \\
National Basic Research Program of China (973 Program) & 49 & $3.1 \%$ \\
\hline
\end{tabular}

Source: Authors' calculations based on Scopus data set

Table 8 indicates the top 5 funding sponsors of the documents funded by some institutions in our sample. The National Natural Science Foundation of China leads the way by funding 265 documents, i.e. $16.7 \%$ of total funded publications. National Science Foundation, an independent federal agency created by Congress of the United States, follows the National Natural Science Foundation of China by funding 137 documents or $8.6 \%$ of total publications. It is worth noting that three of the top 5 funding sponsors in Bitcoin-related studies are from China.The Gini index is usually represented graphically via the Lorenz curve, which shows income distribution by plotting the population percentile in which the income on the horizontal axis and cumulative income on the vertical axis. The Gini coefficient can be used as a yardstick to find out the degree of income equality in a population. It can vary from 0 which corresponds to perfect equality to 1 , i.e., perfect inequality. Lorenz curves and Gini coefficients are also used to investigate the concentration of cited articles in specific journals (Chien et al 2018; Rousseau, 2000; Hart and Perlis, 2021). In the y-axis of the Lorenz curve we drawn, there is the cumulative percentage of studies on Bitcoin with published articles from different countries and funders and the cumulative percentage of the continents is on the x-axis. The Gini coefficient is calculated according to the following formula:

$$
\text { Gini }=1-\sum\left(Y_{i+1}+Y_{i}\right)\left(X_{i+1}-X_{i}\right)
$$

$\mathrm{X}$ in the formula represents the cumulative proportion of the continents in the world, and $Y_{i}$ refers to cumulative proportion of the articles published in continent 
i. A Gini coefficient of 0 (zero) indicates that the articles are distributed equally in the regions, while a value of 1 indicates that all articles in the relevant field are published in only one region. Undoubtedly, as in the distribution of income, there is no perfect equality in the distribution of articles to continental regions.

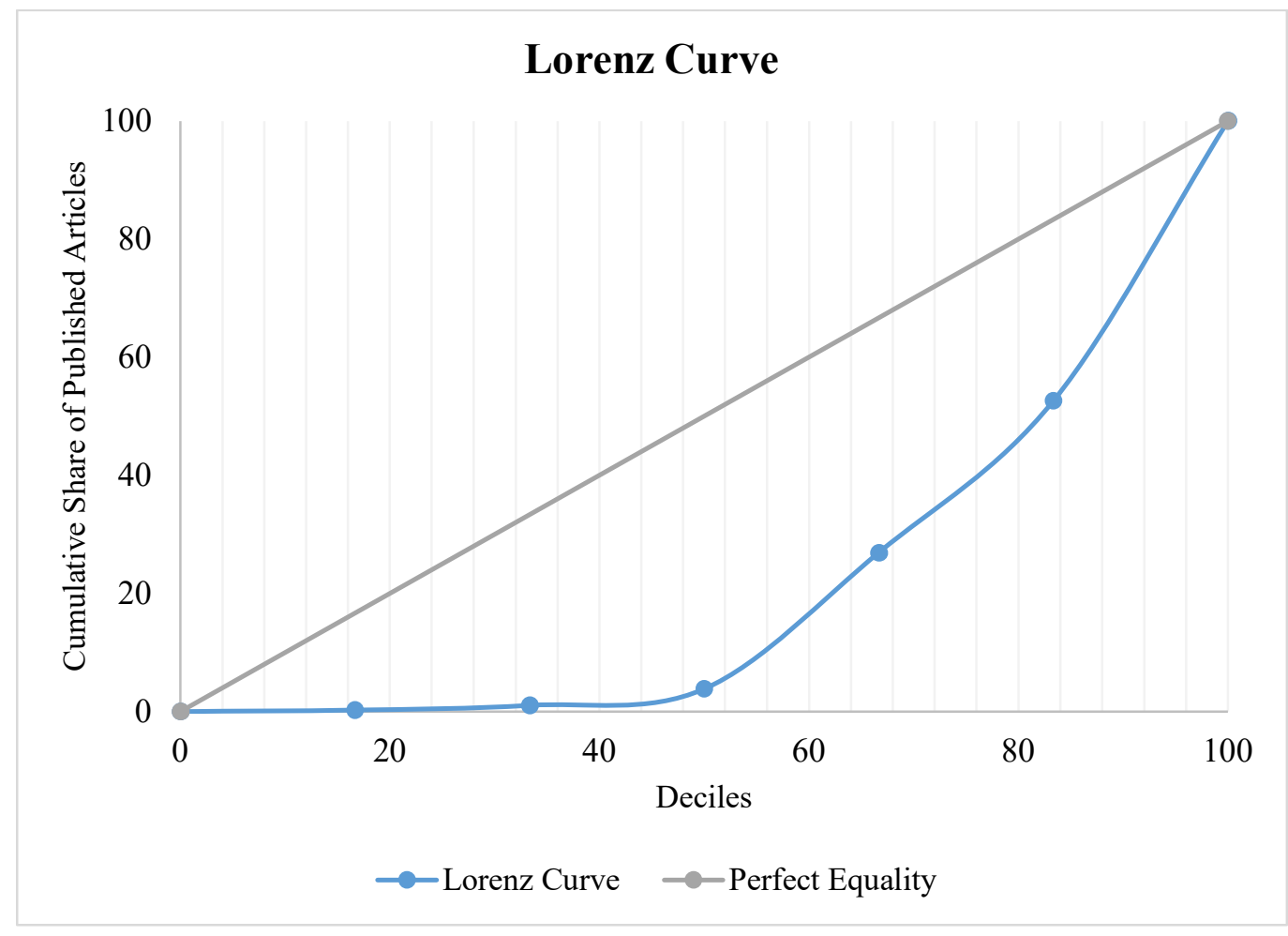

Traditionally, a Gini index of $<0.2$ represents perfect income equality, $0.2-0.3$ relative equality, $0.3-0.4$ adequate equality, $0.4-0.5$ big income gap, and above 0.5 points out to a severe income gap. Therefore, "the warning level" of Gini index is regarded as 0.4. Lorenz curves that are bowed further away from the perfect equality line, which is the diagonal line in the graph, correspond to economies with more income inequality. Similarly, in our graph, we observe a Lorenz curve that is bowed away from the diagonal equality line, which implies a concentration of Bitcoin related academic output in a few continental regions. As Table 8 and Table 9 suggest that there is continental concentration since Chinese and the U.S. institutions, both public and private, invest and provide funds to these studies heavily. Our calculation of Gini index with a value of 0.5514 confirms this interpretation. 
Table 9: Top 10 corresponding author's countries

\begin{tabular}{lcc}
\hline Country & \# of Publications & \% of Total Publications \\
\hline United States & 889 & $15 \%$ \\
China & 573 & $10 \%$ \\
United Kingdom & 413 & $7 \%$ \\
India & 361 & $6 \%$ \\
Germany & 255 & $4 \%$ \\
France & 190 & $3 \%$ \\
Italy & 186 & $3 \%$ \\
Australia & 180 & $3 \%$ \\
Canada & 174 & $3 \%$ \\
South Korea & 153 & $3 \%$ \\
\hline
\end{tabular}

Source: Authors' calculations based on Scopus data set

Some of the studies in our sample, as expected, are carried out by more than one author (possibly from different countries). Therefore, the Scopus database has matched some of the papers with one or more countries. For that reason, 4495 papers that our sample contains, assigned to 5953 countries in total. In Table 9, we showed the top ten corresponding authors' countries. In our sample, the U.S. takes the lead with 889 publications in total and constitutes $15 \%$ of total publications. China and the United Kingdom follow the United States in Bitcoinrelated research. The top ten countries accumulate into $59 \%$ of total publications.

To provide a graphical analysis of the bibliometric data and a visualization of the results, we utilized VOSviewer software (Van Eck \& Waltman, 2010). VOSviewer is a scientific software that collects the data and generates maps based on bibliographic coupling, co-authorship, citation, co-citation and cooccurrence of keywords (Merigo, Cancino, Coronado, \& Urbano, 2016). 


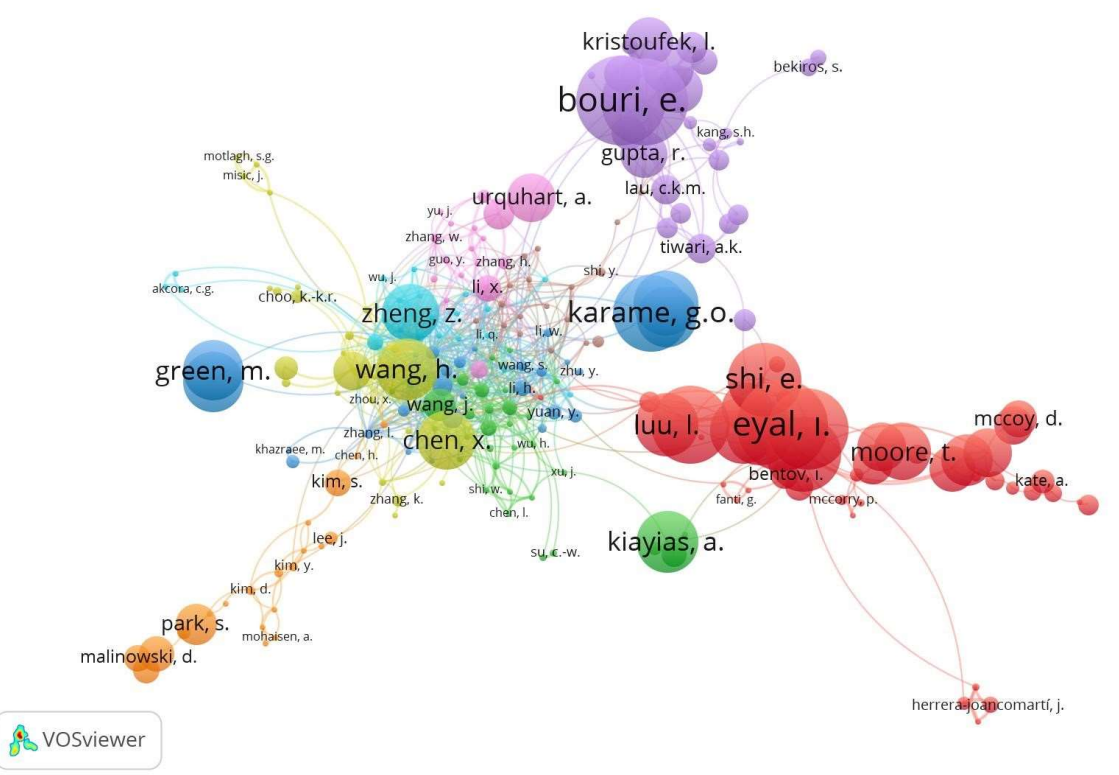

Figure 3: Author network analysis

Figure 3 plots the author network analysis of the documents in our sample. It can be observed there are some prominent authors that are pioneering Bitcoin-related research. Further, there are some clusters indicating potential research collaborations. For example, in the cluster above, we see those researchers whose studies are interconnected are from the field of finance and economics such as Elie Bouri, Andrew Urquhart, Ladislav Kristoufek. On the right side, we see a cluster of researchers mainly from fields such as computer science and engineering. 


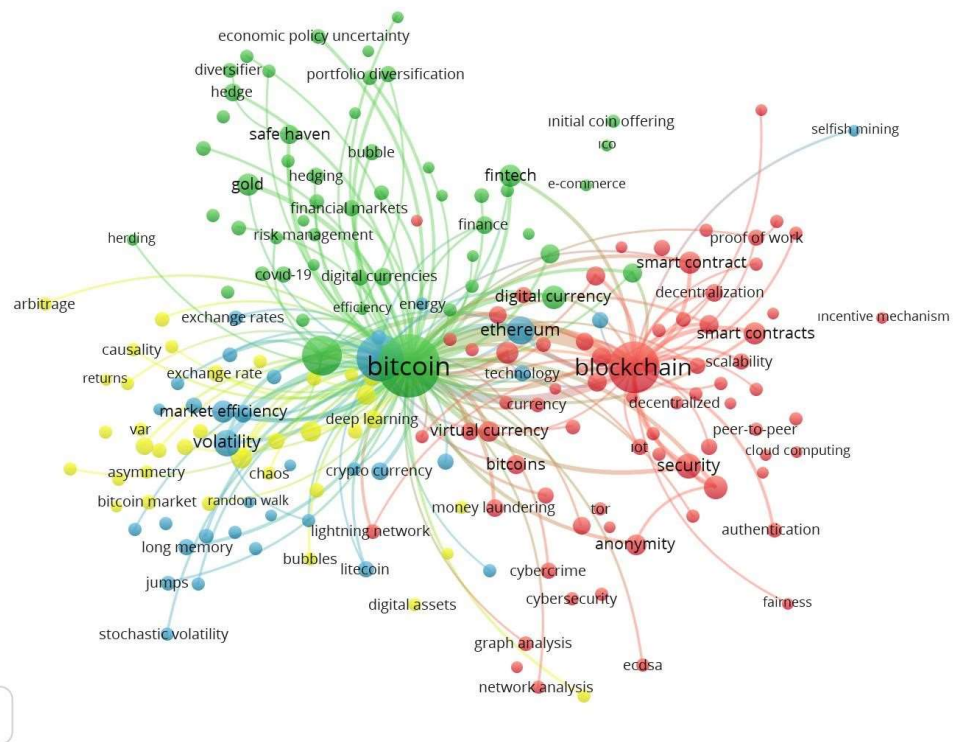

Figure 4: Keyword analysis of articles

Figure 4 represents the network analysis of keywords that appeared in articles in our sample. This cloud map indicates the number of occurrences of words in articles and the relationship of keywords. Findings could be divided into three parts. The red area mainly comprises topics related to blockchain technology and smart contracts. On the other hand, the area that contains the yellow and green clusters includes keywords related to the economics and finance part of Bitcoin. Lastly, the blue area focused on cryptocurrency markets. Not surprisingly, 'Bitcoin' and 'Blockchain' appeared at the center of the network among the two main clusters. Further, interestingly, COVID-19 appeared on the cloud map. This result may show us the increasing academic attention to Bitcoin since COVID-19 is a relatively new phenomenon. 


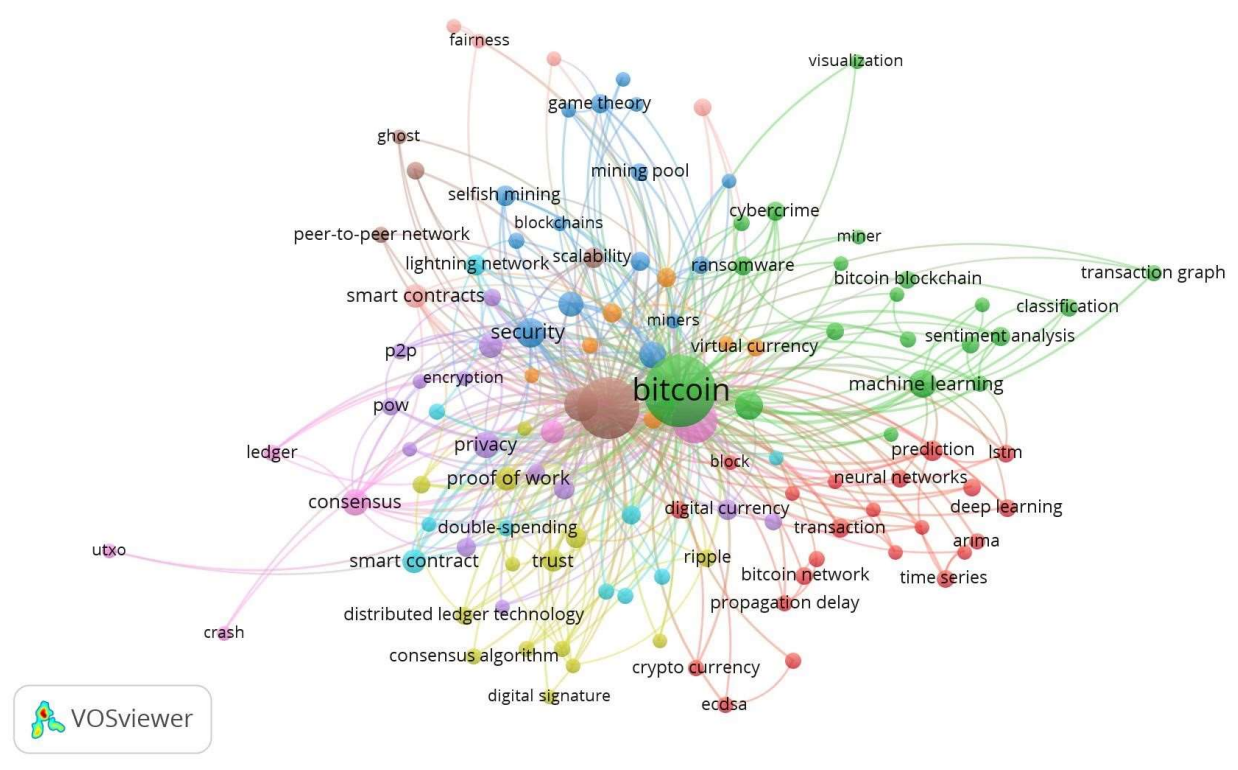

Figure 5: Keyword analysis of conference papers

Figure 5 depicts the cloud map of keywords that appeared in conference papers. Generally, keywords that appeared in the cloud map are similar to that of Figure 4. However, keywords related to economics and finance do not appear as it appears in Figure 4. In Figure 5, the main keywords are related to engineering and computer science, more specifically, to blockchain technology. This result occurs regardless of clusters.

\section{Conclusion}

From a technological point of view, blockchain is a path-breaking paradigm and cryptocurrencies are one of the most important areas in which blockchain technology is used. Among those, Bitcoin is the dominant actor, both in the market capitalization and in the literature interest. In this study, we carried out a comprehensive bibliometric analysis of Bitcoin literature and investigated the growing scientific literature on Bitcoin for the period 2011-2020 by using the Scopus database. Using a sample that includes 4495 documents, we provided insights about dimensions such as the change in the number of publications over the course of years, main research areas, types of published documents, most 
important platforms and sources of Bitcoin publications, highly cited studies, productive authors, author's countries, and finally main funders of Bitcoin-related research. Inspection of the literature from various standpoints also contributed to determining potential research paths in the field. According to findings, the number of publications is skyrocketed, with an over $100 \%$ growth rate, at the initial stages, nevertheless, that trend is declining. Recent developments in the cryptocurrency markets and its usage as a payment method might cause this trend to be reversed, again. Bitcoin-related literature largely comprises research from computer science, $33.4 \%$ of total publications. Engineering, mathematics, and economics follow computer science in the list. Remarkably, we found that three of the top five main institutional sponsors to the Bitcoin-related literature are Chinese. All in all, Bitcoin has opened ample research opportunities in various scientific domains. Therefore, the impacts and future path of Bitcoin and cryptocurrencies needed to be examined more thoroughly. Additionally, recent statements about accepting Bitcoin as a payment method, and its increasing prices might affect the related literature in the upcoming years. However, it is also clear that the future of Bitcoin research will not be limited to any particular discipline. This research was limited to Bitcoin since it is the most used cryptocurrency having the highest market capitalization. Future research should also consider including other cryptocurrencies and using different data sources (Web of Science, SpringerLink, etc.). 


\section{References}

Andrikopoulos, A., Samitas, A., \& Kostaris, K. (2016). Four decades of the journal of econometrics: Coauthorship patterns and networks. Journal of econometrics, 195(1), 23-32.

Akyildirim, E Aysan, AF., Cepni, O., Darendeli SPC. (2021) Do investor sentiments drive cryptocurrency prices?, Economics Letters, 2021

Aysan, AF F Bergigui, M Disli (2021) Blockchain-Based Solutions in Achieving SDGs after COVID-19 Journal of Open Innovation: Technology, Market, and Complexity

Aysan, AF AUI Khan, H Topuz (2021) Bitcoin and altcoins price dependency: Resilience and portfolio allocation in COVID-19 outbreak, Risks.

Aysan, AF AUI Khan, H Topuz A.S. Tunali, (2021) Survival of the fittest: A natural experiment from crypto exchanges, The Singapore Economic Review.

Aysan, AF F Bergigui, M Disli (2021) Using Blockchain-Enabled Solutions as SDG Accelerators in the International Development Space, Sustainability.

Aysan, AF B Sadriu, H Topuz (2020) Blockchain Futures In Cryptocurrencies, Trade And Finance: A Preliminary Assessment, Buletin Ekonomi Moneter Dan Perbankan.

Aysan, AF E Demir, G Gozgor. (2019). Effects of the geopolitical risks on Bitcoin returns and volatility, Research in International Business and Finance.

Bonilla, C. A., Merigó, J. M., \& Torres-Abad, C. (2015). Economics in Latin America: a bibliometric analysis. Scientometrics, 105(2), 1239-1252.

Bouri, E., Molnár, P., Azzi, G., Roubaud, D., Hagfors, L.I. (2017a). On the hedge and safe haven properties of Bitcoin: Is it really more than a diversifier? Finance Research Letters. Volume 20. Pages 192-198, ISSN 1544-6123. https://doi.org/10.1016/j.frl.2016.09.025

Bouri, E., Gupta, R., Tiwari, A.K., Roubaud, D., (2017b). Does Bitcoin hedge global uncertainty? Evidence from wavelet-based quantile-in-quantile regressions. Finance Research Letters. Volume 23. Pages 87-95, ISSN 1544-6123. https://doi.org/10.1016/j.frl.2017.02.009 
Bouri E., Shahzad, S.J.H., Roubaud, D., Kristoufek, L., Lucey, B. (2019). Is Bitcoin a better safe-haven investment than gold and commodities? International Review of Financial Analysis. Volume 63. Pages 322-330, ISSN 1057-5219. https://doi.org/10.1016/j.irfa.2019.01.002

Castillo-Vergara, M., Alverez-Marin, A., Placencio-Hidalgo D. (2018). A bibliometric analysis of creativity in the field of business economics. Journal of Business Research. Volume 85. Pages 1-9, ISSN 0148-2963. https://doi.org/10.1016/j.jbusres.2017.12.011

Chien, T. W., Chow, J. C., Chang, Y., \& Chou, W. (2018). Applying Gini coefficient to evaluate the author research domains associated with the ordering of author names: A bibliometric study. Medicine, 97(39), e12418. https://doi.org/10.1097/MD.0000000000012418

Costa, D. F., Carvalho, F. d. M., \& Moreira, B. C. d. M. (2019). Behavioral economics and behavioral finance: a bibliometric analysis of the scientific fields. Journal of Economic Surveys, 33(1), 3-24.

Docampo, D., \& Cram, L. (2019). Highly cited researchers: a moving target. Scientometrics, 118(3), 1011-1025.

England, C., \& Fratrik, C. (2018). Where to bitcoin? Journal of Private Enterprise, 33(1).

Firdaus, A., Ab Razak, M. F., Feizollah, A., Hashem, I. A. T., Hazim, M., \& Anuar, N. B. (2019). The rise of "blockchain": Bibliometric analysis of blockchain study. Scientometrics, 120(3), 1289-1331.

Guo, Y.-M., Huang, Z.-L., Guo, J., Guo, X.-R., Li, H., Liu, M.-Y., ... Nkeli, M. J. (2021). A bibliometric analysis and visualization of blockchain. Future Generation Computer Systems, 116, 316-332.

Hart, K. L., \& Perlis, R. H. (2021). Authorship inequality: a bibliometric study of the concentration of authorship among a diminishing number of individuals in high-impact medical journals, 2008-2019. BMJ open, 11(1), e046002. https://doi.org/10.1136/bmjopen-2020-046002

Hayes, A. S. (2017). Cryptocurrency value formation: An empirical study leading to a cost of production model for valuing bitcoin. Telematics and Informatics, 34(7), 1308-1321. 
Helbing, P. (2019). A review on IPO withdrawal. International Review of Financial Analysis, 62, 200-208.

Holub, M., \& Johnson, J. (2017). Mapping bitcoin's influence on academic research. Available at SSRN 3094492.

Iefremova, O., Wais, K., \& Kozak, M. (2018). Biographical articles in scientific literature: analysis of articles indexed in Web of Science. Scientometrics, 117(3), 1695-1719.

Khan, M. A., \& Salah, K. (2018). IOT security: Review, blockchain solutions, and open challenges. Future Generation Computer Systems, 82, 395- 411.

Korom, P. (2019). A bibliometric visualization of the economics and sociology of wealth inequality: a world apart? Scientometrics, 118(3), 849-868.

Koskinen, J., Isohanni, M., Paajala, H., Jääskeläinen, E., Nieminen, P., Koponen, H., ... Miettunen, J. (2008). How to use bibliometric methods in evaluation of scientific research? an example from Finnish schizophrenia research. Nordic journal of psychiatry, 62(2), 136-143.

Lee, D. H. (2019). Predictive power of conference-related factors on citation rates of conference papers. Scientometrics, 118(1), 281-304.

Liu, H., Zhang, Y., \& Yang, T. (2018). Blockchain-enabled security in electric vehicles cloud and edge computing. IEEE Network, 32(3), 78-83.

Liu, J., et al. (2016). Bitcoin literature: a co-word analysis. In 6th economics \& finance conference, OECD Paris.

Meiklejohn, S., Pomarole, M., Jordan, G., Levchenko, K., McCoy, D., Voelker, G. M., \& Savage, S. (2013). A fistful of bitcoins: characterizing payments among men with no names. In Proceedings of the 2013 conference on internet measurement conference (pp. 127-140).

Merediz-Solà, I., \& Bariviera, A. F. (2019). A bibliometric analysis of bitcoin scientific production. Research in International Business and Finance, 50, 294-305.

Merigó, J. M., Cancino, C. A., Coronado, F., \& Urbano, D. (2016). Academic research in innovation: a country analysis. Scientometrics, 108(2), 559-593. 
Nadler, P., \& Guo, Y. (2020). The fair value of a token: How do markets price cryptocurrencies? Research in International Business and Finance, 52, 101108.

Nakamoto, S. (2008) Bitcoin: A Peer-to-Peer Electronic Cash System. https://bitcoin.org/bitcoin.pdf

Price, D (1965). Networks of Scientific Papers. Science, 149(3683), 510-515. Retrieved April 9, 2021, from http://www.jstor.org/stable/1716232Ramona, O., Cristina, M. S., \& Raluca, S. (2019). Bitcoin in the scientific literature-a bibliometric study. Studies in Business and Economics, 14(3), 160-174.

Ramona,O.,Cristina,M. \& Raluca,S.(2019).Bitcoin in the Scientific Literature A Bibliometric Study. Studies in Business and Economics, 14(3) 160-174. https://doi.org/10.2478/sbe-2019-0051

Rousseau, R. (2000). Concentration and evenness measures as macro-level scientometric indicators. In: J.Gua-hua (Ed.), Research and university evaluation (pp. 72-89). Beijing: Red Flag Publishing House. (In Chinese). English version is available on http://users.telenet.be/ronald.rousseau/Rousseau_Shanghai 2000.pdf

Sasson, E. B., Chiesa, A., Garman, C., Green, M., Miers, I., Tromer, E., \& Virza, M. (2014). Zerocash: Decentralized anonymous payments from bitcoin. In 2014 IEEE symposium on security and privacy (pp. 459-474).

Tschorsch, F., \& Scheuermann, B. (2016). Bitcoin and beyond: A technical survey on decentralized digital currencies. IEEE Communications Surveys \& Tutorials, 18(3), 2084-2123.

Van Eck, N. J., \& Waltman, L. (2010). Software survey: VOSviewer, a computer program for bibliometric mapping. Scientometrics, 84(2), 523-538.

Wei, G. (2019). A bibliometric analysis of the top five economics journals during 2012-2016. Journal of Economic Surveys, 33(1), 25-59.

Yli-Huumo, J., Ko, D., Choi, S., Park, S., \& Smolander, K. (2016). Where is current research on blockchain technology? - a systematic review. PloS one, 11(10), e0163477. 
Zamore, S., Ohene Djan, K., Alon, I., \& Hobdari, B. (2018). Credit risk research: Review and agenda. Emerging Markets Finance and Trade, 54(4), 811835.

Zyskind, G., Nathan, O., et al. (2015). Decentralizing privacy: Using blockchain to protect personal data. In 2015 IEEE security and privacy workshops (pp. 180-184). 